\title{
Three Mathematical Models for a Integrated Lot Sizing and Scheduling Problem
}

\author{
Socorro Rangel, Michelli Maldonado, \\ Depto de Matemática Aplicada, IBILCE, UNESP, \\ 15054-000, São José do Rio Preto, SP \\ E-mail:socorro@ibilce.unesp.br, michellimaldo@gmail.com
}

\begin{abstract}
The objective of this work is to propose mathematical models for the Integrated Lot Sizing and Scheduling Problem (ILSP) considering a production process involving one stage, one machine and considering sequence dependent set up times and costs. An ilustrative example is used to study the computational behavior of the models when the instances are solved by a general purpose software.
\end{abstract}

Palavras-chave: mixed integer otimization, mathematical formulation, lotsizing, sequence dependent set up

\section{Introduction}

In a manufacturing environment, production planning and scheduling deal with critical decisions such as lot sizing and sequencing of multiple products on capacity-constrained resources. Specifically, these decisions are concerned with determining the production quantities, production timing, and production order of the individual items to meet the estimated demand requirements over the planning horizon. Most practical scheduling problems involve setup times or costs. Because of their importance to the industry and because of the challenges they present to solution methodologies, lot sizing and scheduling problems that involve a sequence dependent setup have attracted attention from many researchers.

Standard models for the lot sizing and for the scheduling problems have been increasingly refined to integrate lot sizing and scheduling decisions [3]. Integrated models have been proposedfor example, by [1] in the glass container industry, [8] for the animal feed supplements industry, and [4] for the soft drink industry. Two main strategies have been used to model the scheduling decisions. The first one is a small bucket strategy based on the GLSP model ((General Lotsizing and Scheduling Problem) [5]. The second strategy is a big bucket one and to obtain the production sequence, constraints based on the asymmetric traveling salesman problem (ATSP) are added to the lot sizing formulation.

The objective of this work is to propose mathematical models for the Integrated Lot Sizing and Scheduling Problem (ILSP) in a general context. In Section 2 a brief description of the production process used as a base for the mathematical models is presented. The mathematical models are presented in Section 3. Section 4 presents an illustrative example and final remarks.

\section{Description of a production planning process}

We will consider a production process in which a set of items are to be produced over a finite planning horizon divided into several discrete periods. Items demands are known in advance and are given for each period. Machine capacities should be taken into account in every period. Moreover, machine changeover costs and times should be taken into account since the setup cost and time is sequence dependent. To prevent infeasilbilities due to machine capacity we will 
allow backorders in each period. The lot scheduling problem considered is then to define which items and the respective lot sizes should be produced in each period. If more them one item is produced in the same period, the production sequence should be also defined. The decisons should be taken considering a minimization of overall production costs computed in terms of inventory, backorder and change over costs.

\section{Three representations for the ILSP}

In this section we describe three mathematical models to represent the one stage (1S), one machine (1M) ILSP described in Section 2. The first model is based on the small bucket approach and the other two are based on the big bucket approach.

\subsection{Small Bucket Model - The GLS1S1M Model}

The GLS1S1M is a lot scheduling model for the one stage, one machine lot scheduling problem described in Section 2 based on the GLSP model in [5]. It contains the usual balance and capacity constraints used to model a capacitated lot sizing problem. To obtain the production sequence, each period of the planning horizon (called macro-period) is divided into a number of microperiods representing the maximum number of setups in each period. The micro-period size is flexible and depends on the item lot size. It is a small bucket approach in the sense that only one item can be produced in each micro-period. Let the following parameters define the problem size:

- $J$ is the number of items;

- $T$ is the number of periods in the planning horizon;

- $N_{t}$ as the number of micro-periods in period $t$.

Let $(i, j, t)$ be the index set defined as: $i, j \in\{1, \ldots, J\} ; t \in\{1, \ldots, T\}$ and $\tau \in N_{t}$ be used to index each micro-period. The data and variables used to represent the problem are described in Table 1.

\begin{tabular}{r|l}
\hline Data Name & Meaning \\
\hline$p_{j}$ & machine capacity needed for production one lot of item $j ;$ \\
$b_{i j}$ & machine capacity needed to changeover from item $i$ to $j ;$ \\
$d_{j t}$ & demand for item $j$ in period $t ;$ \\
$g_{j}$ & backorder cost for item $j ;$ \\
$h_{j}$ & inventory cost for item $j ;$ \\
$I_{j 0}^{+}$ & initial inventory for item $j ;$ \\
$I_{j 0}^{-}$ & initial backorder for item $j ;$ \\
$c_{t}$ & machine capacity in period $t ;$ \\
$s_{i j}$ & machine changeover cost from item $i$ to $j ;$ \\
$N_{t}$ & maximum number of setups (micro periods) in period $t ;$ \\
\hline$I_{j t}^{+}$ & Meaning \\
$I_{j t}^{-}$ & backorders for item $j$ at the end of period $t ;$ \\
$x_{j t \tau}$ & production quantity of item $j$ in micro period $\tau ;$ \\
$z_{i j t \tau}$ & $=1$ if there is a machine changeover from item $i$ to item $j$ in micro period $\tau$. \\
$y_{j t \tau}$ & $=1$ if the machine is setup for item $j$ in micro period $\tau ;$ \\
\hline
\end{tabular}

Table 1: Data and variables for the GLS1S1M Model 
The optimization criterion (1) is to minimize the overall costs taking into account inventory, backorder and machine changeover costs.

$$
\operatorname{Min} \mathrm{Z}=\sum_{j \in J} \sum_{t \in T}\left(h_{j} I_{j t}^{+}+g_{j} I_{j t}^{-}\right)+\sum_{t \in T} \sum_{\tau \in N_{t}} \sum_{i \in J} \sum_{\substack{j \in J \\ j \neq i}} s_{i j} z_{i j t \tau}
$$

The lotsizing constraints are defined by constraints (2)-(7). Constraints (2) represent the balance among demand, production, inventories and backordersfor each item in each time period. Constraints (3) represent the machine capacity in each time period. Constraints (4) guarantee that there is production of item $j$ in micro-period $\tau$ only if the machine is prepared. Constraints (7) controls that, in each micro-period $\tau$ there is at most one changeover.

$$
\begin{aligned}
& I_{j(t-1)}^{+}+I_{j t}^{-}+\sum_{\tau \in N_{t}} x_{j t \tau}-I_{j t}^{+}-I_{j(t-1)}^{-}=d_{j t}, \quad \forall j, \forall t \\
& \sum_{\tau \in N_{t}} \sum_{j \in J} p_{j} x_{j t \tau}+\sum_{\tau \in N_{t}} \sum_{i \in J} \sum_{j \in J} b_{i j} z_{i j t \tau} \leq c_{t}, \quad \forall t \\
& x_{j t \tau} \leq \frac{c_{t}}{p_{j}} y_{j t \tau}, \quad \forall j, \forall \tau \in N_{t}, \forall t \\
& \sum_{j \in J} y_{j t \tau}=1, \quad \forall \tau \in N_{t}, \forall t \\
& z_{i j t \tau} \geq y_{i t(\tau-1)}+y_{j t \tau}-1, \quad \forall \tau \in N_{t}, \forall t, \forall j, i ; i \neq j \\
& \sum_{i \in J} \sum_{\substack{j \in J \\
j \neq i}} z_{i j t \tau} \leq 1 \\
& \forall \tau \in N_{t}, \forall t
\end{aligned}
$$

\subsection{Big Bucket Models: MTZ1S1M and MMC1S1M}

The second strategy is a big bucket one and allows the production of several items in a given period. The decisions associated with lot sizing are based on the Capacitated Lot Sizing Problem (CLSP) (e.g. [5]). To obtain the production sequence in each period the assignment constraints and the subtour elimination constraints proposed for the ATSP are used to guarantee that each item produced in a period is sequenced and to eliminate subsequences respectively. There are several different formulations for the ATSP proposed in the literature. Theses formulations differ mainly on the strategy used to represent the subtour elimination constraints [7]. The date and variables used to define the models are described in Tables 1 and 2.

\begin{tabular}{r|l}
\hline Data Name & Meaning \\
\hline$S_{t}$ & maximum number of tank setups in perÁnod $t ;$ \\
\hline Variable Name & Meaning \\
\hline$I_{j t}^{+}$ & inventory for item $j$ at the end of period $t ;$ \\
$I_{j t}^{-}$ & backorders for item $j$ at the end of period $t ;$ \\
$x_{j t}$ & production quantity of item $j$ in period $t ;$ \\
$z_{i j t}$ & changeover on machine from item $i$ to item $j$ in period $t$. \\
$u_{j t}$ & auxiliary variable - might be used to indicate the production order of item \\
& $j$ in period $t ;$
\end{tabular}

Table 2: Data and variables for the MTZ1S1M and MC1S1M Models.

The optimization criterion (8) is to minimize the overall costs taking into account inventory, backorder and machine changeover costs.

$$
\operatorname{Min} \mathrm{Z}=\sum_{j \in J} \sum_{t \in T}\left(h_{j} I_{j t}^{+}+g_{j} I_{j t}^{-}\right)+\sum_{t \in T} \sum_{i \in J} \sum_{\substack{j \in J \\ j \neq i}} s_{i j} z_{i j t}
$$


The lotsizing constraints are defined by constraints (9)-(11). Constraints (9) and (10) are similar to constraints (??) and (??) from model GLS1S1M. Constraints (11) guarantee that there is production of item $j$ only if the machine is prepared. Note that the setup variable is considered implicitly in terms of the changeover variables and that production may not occur although the machine might be prepared.

$$
\begin{array}{lr}
I_{j(t-1)}^{+}+I_{j t}^{-}+x_{j t}-I_{j t}^{+}-I_{j(t-1)}^{-}=d_{j t}, & \forall j, \forall t \\
\sum_{j \in J} p_{j} x_{j t}+\sum_{i \in J} \sum_{\substack{j \in J \\
j \neq i}} b_{i j} z_{i j t} \leq C_{t}, & \forall t \\
x_{j t} \leq \frac{C_{t}}{p_{j t}}\left(\sum_{\substack{i=i_{0} \\
i \neq j}}^{J} z_{i j t}\right), & \forall j, \forall t
\end{array}
$$

Constraints (12)-(15) model the sequence in which the items will be produced in a given period $t$. They are based on the ATSP formulations. Constraints (12) consider that in each period the machine is initially setup for a ghost item $i_{0}$. The changeover costs associated with the ghost item are zero and do not interfere in the total solution cost. Constraints (13) ensure that if there is a changeover from an item $i$ to any item $k$ then there is a changeover from that item $k$ to an item $j$. Constraints (14) guarantee that each item $j$ is produced at maximum once in each period $t$.

Constraints (12) and (13) alone might generate disconnected subsequences, and thus do not guarantee a proper sequence of the items. The subsequences elimination constraints (15) avoid this situation. Finally constraints (16) define the variables' domain.

$$
\begin{array}{lr}
\sum_{j \in J} z_{i_{0} j t} \geq \sum_{i=i_{0}}^{J} z_{i k t}, & \forall k \in J ; k \neq i, \forall t \\
\sum_{\substack{i=i_{0} \\
i \neq k}}^{J} z_{i k t}=\sum_{\substack{j=i_{0} \\
j \neq k}}^{J} z_{k j t}, & \forall k \in J, \forall t \\
\sum_{\substack{j=i_{0} \\
j \neq i}}^{J} z_{i j t} \leq 1, & \\
\text { Subsequence elimination constraints } & \\
x_{j t} \geq 0, z_{i j t}=0 / 1, & \forall i=i_{0}, 1, \ldots, J, \forall t \\
\end{array}
$$

\section{The MTZ1S1M Model}

The first bigbucket model for the one stage one machine ILSP is based on the subtour elimination constraints given by Miller Miller, Tucker and Zemlin (1960) who introduced such constraints for a vehicle routing problem where each route was constrained to have no more than a certain number of clients. A new set of auxiliary variables:

- $u_{j t}$

are necessary to model constraints (15) based on the Miller, Tucker and Zemlin proposal:

$$
u_{j t} \geq u_{i t}+1-(J)\left(1-z_{i j t}\right) ; \quad \forall i, \forall j ; i \neq j ; \forall t
$$

The MTZ1S1M is then defined by the objective function (8), the lotsizing constraints (9) - (16), the assignment constraints (9) - (16), the subsequence elimination constraint (17) and the domain 
constraints (19).

$$
x_{j t} \geq 0, u_{j t} \geq 0, z_{i j t}=0 / 1 \quad \forall i, j ; \forall t .
$$

\section{The MMC1S1M Model}

The MTZ constraints used to eliminate subsequences, (17), in the model MTZ1S1M are of polynomial order, thus allowing their inclusion a priori. However, a formulation based on this constraints provides a weak linear relaxation dual bound. To streghen the formulation the multicommodity-flow formulation for the ATSP is used to replace constraints (15). To obtain the second model based on the bigbucket strategy (Model MMC1S1M) we need to define:

- index $r \in\{1, \ldots, J\}$;

- $m_{\text {rijt }}$.

The idea behind this formulation is that there are $J$ commodities available at node $i_{0}$ and a demand of one unit of commodity $j$ at node $j$. If $m_{\text {rijt }}=1$ then the flow of commodity $r$ flows from node $i_{0}$ no node $r$ through arc $(i, j)$. In terms of the items sequence in period $t$, it means that if product $r$ is included in the production sequence, then product $j$ follows product $i$ in such sequence.

The constraints (20)-(23) eliminates disconnected subsequence of items. Since only the items which are produced (i.e. $x_{r t}>0$ )should be sequenced, constraints (20) and (21) take place only when the machine is prepared for item $r$. These constraints guarantee that if product $r$ is included in the sequence at least one other item should be also included.

$$
\begin{array}{ll}
\sum_{j \in J} m_{r i_{0} j t}-\sum_{j \in J} m_{r j i_{0} t}=\sum_{\substack{j=i_{0} \\
j \neq r}}^{J} z_{j r t}, & \forall r, \forall t \\
\sum_{\substack{j=i_{0} \\
j \neq r}}^{J} m_{r j r t}-\sum_{\substack{j=i_{0} \\
j \neq r}}^{J} m_{r r j t}=\sum_{\substack{j=i_{0} \\
j \neq r}}^{J} z_{j r t}, & \forall r, \forall t
\end{array}
$$

Constraints (22) are the flow conservation constraints, for all but product $r$ in node $r$. And constraints (23) states that item $j$ should follow item $i$ in the sequence that includes item $r$ only if there is a changeover from product $i$ to product $j$.

$$
\begin{array}{lr}
\sum_{\substack{i=i_{0} \\
i \neq j}}^{J} m_{r i j t}=\sum_{\substack{i=i_{0} \\
i \neq j}}^{J} m_{r j i t}, & \forall r, \forall j ; j \neq r, \forall t \\
m_{r i j t} \leq z_{i j} & \forall i, j=i_{0}, 1, \ldots, J ; \forall r ; \forall t
\end{array}
$$

The multi-commodity-flow model for the single stage single machine lot scheduling problem (MM1S1M) is defined by the objective function (8), the constraints (9)-(14), the subtour elimination constraints (20)-(23), and the domain constraints (24).

$$
x_{j t} \geq 0, m_{\text {rijt }} \geq 0, z_{i j t}=0 / 1 \quad \forall i, j, r ; \forall t .
$$

\section{An ilustrative example}

A computational experiment using an ilustrative example adapted from [1], was conducted to compare the GLS1S1M, the MTZ1S1M and the MMC1S1M models. The example considers three products and three periods The models were implemented using the AMPL modelling language [2] and solved by the CPLEX 12.1 [6]. 
This instance of the GLS1S1M model have 208 variables and 132 constraints, the MTZ1S1M instance have 90 variables and 96 constraints, and the MMC1S1M instance have 225 variables and 204 constraints. The objective value is 787 . The two bigbucket models had a similar computational behavior. Both were solve in the root node of the Cplex Branch and Cut tree after the inclusion of 17 cutting planes. To solve the instance of the GLS1S1M model it was necessary to examine 450 nodes in the branch and bound tree and 19 cutting planes. The time necessary to solve the linear relaxations of the GLS1S1M and MMC1S1M was similar, it was higher than the time necessary to solve the linear relaxation of the MTZ1S1M instance. A computational study is being prepared to evaluate what is the influence of the formulations when large scale instances of the 1S1MILSP are solved by genral purpose software.

\section{Acknowledgements}

This work was partially funded by the Brazilian agencies FAPESP, CNPq and CAPES.

\section{References}

[1] B. Almada-lobo, D. Klabjan, M. Antónia carravilla, and J. F. Oliveira. Single machine multi-product capacitated lot sizing with sequence-dependent setups. International Journal of Production Research, 45(20):4873-4894, 2007.

[2] AMPL. A modeling languagem for mahtematical programming. See www.ampl.com (09/08/2012), 2012.

[3] A. Clark, B. Almada-Lobo, and C. Almeder. Lot sizing and scheduling: industrial extensions and research opportunities. International Journal of Production Research, 49(9):2457-2461, 2011.

[4] D. Ferreira, R. Morabito, and S. Rangel. Relax and fix heuristics to solve one-stage onemachine lot-scheduling models for small-scale soft drink plants. Computers $\&$ Operations Research, 37(4):684-691, Apr. 2010.

[5] B. Fleischmann and H. Meyr. The general lotsizing and scheduling problem. OR Spectrum, 19(1):11-21, 1997.

[6] IBM. ILOG - CPLEX 12.1 - mathematical programming optimizers. See www.ibm.com (09/08/2012), 2012.

[7] T. Oncan, A. K. Altinel, and G. Laporte. A comparative analysis of several asymmetric traveling salesman problem formulations. Computers \& Operations Research, 36(3):637-654, Mar. 2009.

[8] E. A. Toso, R. Morabito, and A. R. Clark. Lot sizing and sequencing optimisation at an animal-feed plant. Computers $\mathcal{G}$ Industrial Engineering, 57(3):813-821, Oct. 2009. 